\title{
Exponential Stability of Stochastic Nonlinear Dynamical Price System with Delay
}

\author{
Wenli Zhu, ${ }^{1}$ Xinfeng Ruan, ${ }^{1}$ Ye Qin, ${ }^{2}$ and Jie Zhuang ${ }^{3}$ \\ ${ }^{1}$ School of Economic Mathematics, Southwestern University of Finance and Economics, Chengdu 611130, China \\ ${ }^{2}$ Teaching Research Training Center in Xindu District of Chengdu, Chengdu 610500, China \\ ${ }^{3}$ Sichuan University of Science and Engineering Library, Zigong 643000, China
}

Correspondence should be addressed to Wenli Zhu; zhuwl@swufe.edu.cn

Received 30 January 2013; Revised 28 April 2013; Accepted 17 May 2013

Academic Editor: Wuquan Li

Copyright (C) 2013 Wenli Zhu et al. This is an open access article distributed under the Creative Commons Attribution License, which permits unrestricted use, distribution, and reproduction in any medium, provided the original work is properly cited.

Based on Lyapunov stability theory, Itô formula, stochastic analysis, and matrix theory, we study the exponential stability of the stochastic nonlinear dynamical price system. Using Taylor's theorem, the stochastic nonlinear system with delay is reduced to an $n$-dimensional semilinear stochastic differential equation with delay. Some sufficient conditions of exponential stability and corollaries for such price system are established by virtue of Lyapunov function. The time delay upper limit is solved by using our theoretical results when the system is exponentially stable. Our theoretical results show that if the classical price Rayleigh equation is exponentially stable, so is its perturbed system with delay provided that both the time delay and the intensity of perturbations are small enough. Two examples are presented to illustrate our results.

\section{Introduction}

Let us make the following assumptions.

(H1.1) Demand for product is quadratic function with respect to price.

(H1.2) The price is not very sensitive to the change of inventory. That is, damping of nonlinear dynamical system $\lambda$ is a $\varepsilon$-order infinitesimal ( $\varepsilon>0$ is small enough).

(H1.3) Stochastic noise is related to price. That is, it can be treated as Gaussian white noise, and the excitation coefficient is $\sqrt{\varepsilon}$-order infinitesimal.

The price system can be described by linear equations because of their convenience in mathematical treatment. Therefore linear equations play an important role in theory and their applications. However, they can not perfectly describe the process of the price fluctuation in nonlinear version. Then the nonlinear equations should be employed, for their virtues that can deeply reflect the rules of price fluctuation.

Suppose $S(t), D(t), P(t)$, and $Q(t)$ as supply, demand, price, and inventory at time $t$, respectively. $S_{0}$ is initial supply, $a, b, c, \lambda, \alpha$ and $\gamma$ are constants, and alphabet with a bar is equilibrium value. Reference [1] gives a deterministic nonlinear price model as follows:

$$
\frac{d^{2} P}{d t^{2}}=\lambda\left(a P^{2}+b P+c\right) \frac{d P}{d t}-\lambda \alpha P-\lambda\left(S_{0}-\bar{D}\right) .
$$

If there exists an equilibrium point in the above price system, denoted by $\bar{P}$, then $\bar{P}=\left(\bar{D}-S_{0}\right) / \alpha$ and $\bar{D}=S_{0}+\alpha \bar{P}$. Let $x(t)=P(t)-\bar{P}$ and $t=\eta / \sqrt{\alpha \lambda}, \mu=\sqrt{\lambda / \alpha}$. Applying Lienard transformation, we obtain the classical price Rayleigh equation as follows:

$$
\begin{gathered}
\frac{d x}{d \eta}=-y+\varepsilon \widetilde{\mu}\left(\frac{1}{3} a x^{3}+\frac{1}{2} b_{0} x^{2}+c_{0} x\right), \\
\frac{d y}{d \eta}=x,
\end{gathered}
$$

where $b_{0}=2 a \bar{P}+b, c_{0}=a \bar{P}^{2}+b \bar{P}+c, \mu \triangleq \varepsilon \widetilde{\mu}$.

For many real-world systems, there always exist random disturbances such as the measurement error and the control input of the system [2-5]. The basic source of random 
disturbance is Gaussian white noise, which represents the joint effects of a large number of independent random forces acting on the systems, and the influence of individual is not significant. By (H1.3), the stochastic nonlinear dynamical price system can be described by stochastic differential equation (SDE for short) as follows [5]:

$$
\begin{gathered}
d x=\sqrt{\lambda \alpha}\left[-y+\varepsilon \tilde{\mu}\left(\frac{1}{3} a x^{3}+\frac{1}{2} b_{0} x^{2}+c_{0} x\right)\right] d t \\
+\varepsilon^{1 / 2} \gamma d W(t) \\
d y=\sqrt{\lambda \alpha} x d t
\end{gathered}
$$

where $\{W(t), t \geq 0\}$ is 1 -dimensional Brownian motion. The above system also can be rewritten as the following matrix form:

$$
d z(t)=f(t, z(t)) d t+h(t, z(t)) d W(t),
$$

where $f: R^{+} \times R^{2} \rightarrow R^{2}, h: R^{+} \times R^{2} \rightarrow R^{2}, f(t, z(t))=$ $\sqrt{\lambda \alpha}\left(-y+\varepsilon \tilde{\mu}\left(a x^{3} / 3+b_{0} x^{2} / 2+c_{0} x\right), x\right)^{T}, z(t)=(x(t), y(t))^{T} \in$ $R^{2}$, and $h(t, z(t))=(\sqrt{\varepsilon} \gamma, 0)^{T}$.

Supply is not only influenced by price and demand but also influenced by production management, information feedback, transportation, and so forth. Therefore, $S(t)$ not only depends on the situation at $t$ but also on the certain period $t-\tau$ ( $\tau>0$ is a given time delay) in the past [6$9]$. Furthermore, the parameter perturbation of the system's internal structure should also be taken into account in this paper.

Based on the abovementioned, the price system (4) can be extended to more general $n$-dimensional stochastic nonlinear price systems with delay as follows:

$$
\begin{gathered}
d x(t)=[f(t, x(t), x(t-\tau))+\bar{f}(t, x(t), x(t-\tau))] d t \\
+h(t, x(t), x(t-\tau)) d W(t), \quad t \geq 0, \\
x(t)=\xi(t), \quad-\tau \leq t \leq 0,
\end{gathered}
$$

where $\tau$ is a given time delay. The maps $f, \bar{f} \in C\left(R^{+} \times\right.$ $\left.R^{n} \times R^{n}, R^{n}\right), h \in C\left(R^{+} \times R^{n} \times R^{n}, R^{n \times m}\right) . \bar{f}$ represents the uncertainty. $\{W(t), t \geq 0\}$ is an $m$-dimensional Brownian motion, and the term $h(t, x(t), x(t-\tau)) d W(t)$ represents the stochastic disturbance. Furthermore, we always assume that $f(t, 0,0)=\bar{f}(t, 0,0)=h(t, 0,0) \equiv 0$ for the stability purpose of this paper.

Stability is a very important dynamical feature for the stochastic price system with delay, and it is one of the main purposes of system designing $[5,6]$. Keeping the price system steady within the cycle as long as possible to avoid inflation or deflation has the vital significance for the healthy development of the economy of the country. There is a rich literature on time delay system and stochastic system. Stability of stochastic system has been studied. See, for example, Liu and Feng [2], Liu and Deng [3], Yong and Zhou [4], Li and $\mathrm{Xu}$ [5], and Mao [10]. Stability of time delay system has been studied. See, for example, Kazmerchuk et al.
[6], Lv and Liu [7], Lv and Zhou [8], Zhu et al. [11], Zhu and Yi [12], and Trinh and Aldeen [13]. Mao [14], Mao and Shah [15], Zhu and $\mathrm{Hu}$ [16], Zhu and $\mathrm{Hu}$ [17], and S. Xie and L. Xie [18] established some stability criteria of the stochastic system with delay by using an LMI approach. The Hopf bifurcation of price Rayleigh delayed equation on deterministic case has been studied extensively in recent years. See, for example, [6-8]. The stability and the optimal control of stochastic nonlinear dynamical price model has been studied in [5]. Unfortunately, there is a little previous literature on stochastic nonlinear dynamical price system with delay. Thus, we aim to fill this gap in this paper. We plug the time delay, the parameter perturbation, and the stochastic item into nonlinear dynamical price system (2). Such models may be identified as stochastic differential delayed equations (SDDEs for short). Our target in this paper is to derive some sufficient conditions of exponential stability for SDDEs.

$\mathrm{Li}$ and $\mathrm{Xu}$ only analyzed the stability for SDEs (4) in virtue of the marginal probability density about $x$ in [5] but did not give the sufficient condition for stability. In this paper, using Taylor's theorem, the $n$-dimensional nonlinear SDDE (5) is reduced to an $n$-dimensional semilinear SDDE correspondingly. Some sufficient conditions of exponential stability and corollaries for such price system are established by using Lyapunov function. The time delay upper limit is solved by using our theoretical results when the system is exponentially stable. Thus, [5] is promoted and improved. Our theoretical results show that if the classical price Rayleigh equation (2) is exponentially stable, so is its perturbed system (5) with delay provided that both the time delay and the intensity of perturbations are small enough. Those results will help our government make a macrocontrol for price system and timely adjust their pricing strategies.

The rest of this paper is organized as follows. In Section 2, we introduce the definition of the exponential stability of SDDEs. Section 3 is devoted to the sufficient conditions for exponential stability and almost surely exponential stability of price system. Section 4 presents two simple examples to illustrate our results. Finally, Section 5 concludes the paper.

\section{Preliminaries}

Throughout this paper and unless specified, we let $W(t)=$ $\left(W_{1}(t), \ldots, W_{m}(t)\right)^{T}$ be an $m$-dimensional Brownian motion defined on a complete probability space $(\Omega, \mathscr{F}, P)$ with a natural filtration $\left\{\mathscr{F}_{t}\right\}_{t \geq 0}$ (i.e., $\mathscr{F}_{t}=\sigma\{W(s): 0 \leq s \leq t\}$ and augmented by all the $P$-null sets in $\mathscr{F})$. Denote by $|\cdot|$ the Euclidean norm. If $A$ is a vector or matrix, its transpose is denoted by $A^{T}$. If $A$ is a matrix, denote by $\|A\|$ the operator norm of $A$, that is, $\|A\|=\sup \{|A x|:|x|=1\}$. $\xi(\cdot) \in C[-\tau, 0]$ is the initial path of $x$, where $\tau>0$ is a given finite time delay and $C[-\tau, 0]$ is the set of continuous functions from $[-\tau, 0]$ into $R^{n}$. Moreover, denote by $L_{\mathscr{F}_{0}}^{p}\left(-\tau, 0 ; R^{n}\right)$ the family of $R^{n}$ valued adapted stochastic processes $\xi(s),-\tau \leq s \leq 0$ such that $\xi(s)$ is $\mathscr{F}_{0}$-measurable and $\int_{-\tau}^{0} E|\xi(s)|^{p} d s<+\infty(p>1)$.

We define a norm mean square in $S^{n}$ as follows:

$$
|x|_{\mathrm{MS}}=\left(E|x|^{2}\right)^{1 / 2}, \quad \text { for any } x \in S^{n}
$$


where $S^{n}$ is the set of random variable in probability space $(\Omega, \mathscr{F}, P)$. Similarly, we define $\|A\|_{\mathrm{MS}}=\sup \left\{|A x|_{\mathrm{MS}}:|x|_{\mathrm{MS}}=\right.$ $1\}$. by

Then, the Itô integral of $h(t, x(t))$ (from $a$ to $b$ ) is defined

$$
\begin{aligned}
& \text { (I) } \int_{a}^{b} h(t, x(t)) d W(t) \\
& \quad=\lim _{\lambda \rightarrow 0} \sum_{i=0}^{N} h\left(t_{i}, x\left(t_{i}\right)\right)\left[W\left(t_{i+1}\right)-W\left(t_{i}\right)\right]
\end{aligned}
$$

(limit in $\left.L^{2}(P)\right)$,

where $h$ is a stochastic process with value in $S^{n \times m}$ and $\int_{a}^{b}\|h(t, x(t))\|_{\mathrm{MS}}^{2} d t<\infty(0 \leq a<b)$. Let $a=t_{0}<t_{1}<$ $\cdots<t_{N}=b, \lambda=\max \left\{\Delta t_{i}, i=0,1, \ldots, N-1\right\}, \Delta t_{i}=t_{i+1}-t_{i}$, and $\lim _{\mathrm{MS}}$ be a limit in mean square sense.

It is proved directly from the definition of Itô integral that

$$
\int_{a}^{b} W(t) d W(t)=\frac{1}{2}\left[W^{2}(b)-W^{2}(a)\right]-\frac{1}{2}(b-a),
$$

where $W(\cdot)$ is 1-dimensional Brownian motion. The extra term $-(b-a) / 2$ shows that the Itô stochastic integral does not behave like ordinary integrals. It leads to Itô-type stochastic system being different from non-Itô-type. See [19] for the details.

Now, let us present an existence and uniqueness result for system (5). First, we make the following assumptions for the coefficients of (5).

(H2.1) The maps $f, \bar{f}$, and $h$ are locally Lipschitz continuous.

(H2.2) The maps $f, \bar{f}$, and $h$ satisfy the linear growth condition.

Theorem 1 (see [4]). Let (H2.1) and (H2.2) hold. Then, for any $\xi(t) \in L_{\mathscr{F}_{0}}^{2}\left(-\tau, 0 ; R^{n}\right)$, (5) has a unique strong solution which is denoted by $x(t ; \xi)$, and $x(t ; \xi)$ is square integrable. So, (5) has a trivial solution $x(t ; 0)=0$.

For stochastic system, exponential stability in mean square and almost surely exponential stability are generally used [2].

Definition 2. The trivial solution of system (5) is said to be $p$ th moment exponentially stable, if there exists a positive constant $\varepsilon$ such that

$$
\limsup _{t \rightarrow \infty} \frac{1}{t} \ln \left(E|x(t ; \xi)|^{p}\right) \leq-\varepsilon
$$

for any $\xi \in L_{\mathscr{F}_{0}}^{p}\left(-\tau, 0 ; R^{n}\right)$, where $-\varepsilon$ is called $p$ th moment Lyapunov exponent of the trivial solution. stable.

In particular, $p=2$; it is called mean square exponentially
Definition 3. The trivial solution of system (5) is said to be almost surely exponentially stable, if there exists a positive constant $\lambda$ such that

$$
\limsup _{t \rightarrow \infty} \frac{1}{t} \ln |x(t ; \xi)| \leq-\lambda \quad \text { a.s. }
$$

for any $\xi \in L_{\mathscr{F}_{0}}^{p}\left(-\tau, 0 ; R^{n}\right)$, where $-\lambda$ is called almost surely Lyapunov exponent of the trivial solution.

\section{Exponential Stability for Stochastic Price System with Delay}

To obtain sufficient condition of the exponential stability of system (5), we assume that the functions $f(t, x, y), \bar{f}(t, x, y)$ are 1-order continuously differentiable in the neighbourhood of $(t, 0,0)$ with respect to $(x, y) \in R^{n} \times R^{n}$. According to Taylor expansion, for $0<\theta<1$

$$
\begin{aligned}
& f(t, x, y)=f_{x}(t, \theta x, \theta y) x+f_{y}(t, \theta x, \theta y) y \\
& \bar{f}(t, x, y)=\bar{f}_{x}(t, \theta x, \theta y) x+\bar{f}_{y}(t, \theta x, \theta y) y .
\end{aligned}
$$

Thus,

$$
\begin{aligned}
f(t, x, y)+\bar{f}(t, x, y) & \\
= & \left(f_{x}(t, \theta x, \theta y)+\bar{f}_{x}(t, \theta x, \theta y)\right) x \\
& +\left(f_{y}(t, \theta x, \theta y)+\bar{f}_{y}(t, \theta x, \theta y)\right) y .
\end{aligned}
$$

Therefore, system (5) can be reduced to an $n$-dimensional semilinear stochastic differential delayed equation as follows:

$$
\begin{gathered}
d x(t)=[f(t, x(t), x(t-\tau))+\bar{f}(t, x(t), x(t-\tau))] d t \\
+h(t, x(t), x(t-\tau)) d W(t), \quad t \geq 0, \\
x(t)=\xi(t), \quad-\tau \leq t \leq 0,
\end{gathered}
$$

where $A_{1}, B_{1}$ are $n \times n$ matrices. $A_{1}(t), A_{2}(t-\tau), B_{1}(t)$, and $B_{2}(t-\tau)$ represent the uncertainties. They are bounded $n \times n$ matrix-valued functions. Here, $\tau, W(t), h(t, x(t), x(t-\tau))$, and $\xi(t)$ are the same as in the previous section.

We make the following assumption for the coefficients of system (13).

(H3.1) There exist nonnegative constants $\alpha_{i}, \beta_{i}(i=1,2,3)$, for any $t \geq 0$ such that

$$
\begin{aligned}
\left\|A_{1}(t)\right\| \leq \alpha_{1}, & \left\|A_{2}(t-\tau)\right\| \leq \alpha_{2}, \\
\left\|B_{1}(t)\right\| \leq \beta_{1}, & \left\|B_{2}(t-\tau)\right\| \leq \beta_{2}
\end{aligned}
$$

and for any $(t, x(t), x(t-\tau)) \in R^{+} \times R^{n} \times R^{n}$ such that

$$
\begin{gathered}
\operatorname{tr}\left[h^{T}(t, x(t), x(t-\tau)) h(t, x(t), x(t-\tau))\right] \\
\leq \alpha_{3}|x(t)|^{2}+\beta_{3}|x(t-\tau)|^{2} .
\end{gathered}
$$


Theorem 4. Let h satisfy (H2.1)-(H2.2), and condition (14) hold. Then, for any $\xi(t) \in L_{\mathscr{F}_{0}}^{2}\left(-\tau, 0 ; R^{n}\right)$, system (13) has a unique strong solution which is denoted by $x(t ; \xi)$, and $x(t ; \xi)$ is square integrable. So, (13) has a trivial solution $x(t ; 0)=0$.

See Mao [10] for the proof of Theorem 4.

In the study of mean square exponential stability, it is often to use a quadratic function as the Lyapunov function; that is, $V(t, x)=x^{T}(t) G x(t)$, where $G$ is a symmetric positive definite $n \times n$ matrix (see $[11,20]$ ).

Theorem 5. Let (H3.1) holds, and then the trivial solution of system (13) is exponentially stable in the mean square. Assume that there exists a pair of symmetric positive definite $n \times n$ matrices $G$ and $Q$ such that

$$
G(A+B)+(A+B)^{T} G=-Q,
$$

$$
\begin{aligned}
& \lambda_{\min }(Q) \\
& >\|G\|\left(2 \alpha_{1}+2 \alpha_{2}+2 \beta_{1}+2 \beta_{2}+\alpha_{3}+\beta_{3}\right)+2\|G B\| \\
& \quad \cdot \sqrt{2 \tau\left[6 \tau\left(\|A\|^{2}+\|B\|^{2}+\alpha_{1}^{2}+\beta_{1}^{2}+\alpha_{2}^{2}+\beta_{2}^{2}\right)+\alpha_{3}+\beta_{3}\right]},
\end{aligned}
$$

where $\lambda_{\min }(Q)>0$ is the smallest eigenvalue of $Q$.

Proof. Fix the initial data $\xi(t)$ arbitrarily, and write $x(t ; \xi)=$ $x(t)$ simply. Applying Itô's formula to $x^{T}(t) G x(t)$, we have

$$
\begin{aligned}
d\left[x^{T}(t)\right. & G x(t)] \\
= & 2 x^{T}(t) G[A x(t)+B x(t-\tau)] d t \\
& +2 x^{T}(t) G\left[A_{1}(t) x(t)+B_{1}(t) x(t-\tau)\right] d t \\
& +2 x^{T}(t) G\left[A_{2}(t-\tau) x(t)+B_{2}(t-\tau) x(t-\tau)\right] d t \\
& +2 x^{T} G h(t, x(t), x(t-\tau)) d W(t) \\
& +\operatorname{tr}\left[h^{T}(t, x(t), x(t-\tau)) G h(t, x(t), x(t-\tau))\right] d t .
\end{aligned}
$$

By (16), we can estimate the first item of (18) as follows:

$$
\begin{aligned}
2 x^{T}(t) & G[A x(t)+B x(t-\tau)] \\
= & 2 x^{T}(t) G[A x(t)+B x(t-\tau)] \\
& +2 x^{T}(t) G B x(t)-2 x^{T}(t) G B x(t) \\
\leq & -\lambda_{\min }(Q)|x(t)|^{2}+\beta|x(t)|^{2} \\
& +\frac{1}{\beta}\|G B\|^{2} \cdot|x(t)-x(t-\tau)|^{2},
\end{aligned}
$$

where

$\beta=\|G B\|$

$$
\cdot \sqrt{2 \tau\left[6 \tau\left(\|A\|^{2}+\|B\|^{2}+\alpha_{1}^{2}+\beta_{1}^{2}+\alpha_{2}^{2}+\beta_{2}^{2}\right)+\alpha_{3}+\beta_{3}\right]} .
$$

By (14), we can estimate the second item and the third item of (18), respectively,

$$
\begin{aligned}
2 x^{T}(t) & G\left[A_{1}(t) x(t)+B_{1}(t) x(t-\tau)\right] \\
\leq & \|G\|\left[\left(2 \alpha_{1}+\beta_{1}\right)|x(t)|^{2}+\beta_{1}|x(t-\tau)|^{2}\right], \\
2 x^{T}(t) G\left[A_{2}(t-\tau) x(t)+B_{2}(t) x(t-\tau)\right] & \\
\leq & \|G\|\left[\left(2 \alpha_{2}+\beta_{2}\right)|x(t)|^{2}+\beta_{2}|x(t-\tau)|^{2}\right] .
\end{aligned}
$$

By (15), the last item of (18) yields

$$
\begin{gathered}
\operatorname{tr}\left[h^{T}(t, x(t), x(t-\tau)) G h(t, x(t), x(t-\tau))\right] \\
\leq\|G\|\left[\alpha_{3}|x(t)|^{2}+\beta_{3}|x(t-\tau)|^{2}\right] .
\end{gathered}
$$

Substituting (21) and (22) into (18), we get

$$
\begin{aligned}
d\left[x^{T}(t)\right. & G x(t)] \\
\leq & -\left[\lambda_{\min }(Q)-\|G\|\left(2 \alpha_{1}+\beta_{1}+2 \alpha_{2}+\beta_{2}+\alpha_{3}\right)-\beta\right] \\
& \cdot|x(t)|^{2} d t+\|G\|\left(\beta_{1}+\beta_{2}+\beta_{3}\right)|x(t-\tau)|^{2} d t \\
+ & \frac{1}{\beta}\|G B\|^{2} \cdot|x(t)-x(t-\tau)|^{2} d t \\
+ & 2 x^{T}(t) G h(t, x(t), x(t-\tau)) d W(t) .
\end{aligned}
$$

If (17) holds, then we can choose $\varepsilon>0$ small enough such that

$$
\begin{aligned}
& \lambda_{\min }(Q) \\
& =\|G\|\left(2 \alpha_{1}+2 \alpha_{2}+\beta_{1}+\beta_{2}+\alpha_{3}+\varepsilon\right) \\
& +\|G\|\left(\beta_{1}+\beta_{2}+\beta_{3}\right) e^{\varepsilon \tau}+\beta \\
& +\frac{1}{\beta}\|G B\|^{2}\left\{2 \tau\left[6 \tau\left(\|A\|^{2}+\alpha_{1}^{2}+\alpha_{2}^{2}\right)+\alpha_{3}\right] e^{\varepsilon \tau}\right. \\
& \left.\quad+2 \tau\left[6 \tau\left(\|B\|^{2}+\beta_{1}^{2}+\beta_{2}^{2}\right)+\beta_{3}\right] e^{2 \varepsilon \tau}\right\} .
\end{aligned}
$$

Applying Itô’s formula to $e^{\varepsilon t} x^{T}(t) G x(t)$, we have

$$
\begin{aligned}
d\left[e^{\varepsilon t} x^{T}(t) G x(t)\right]= & \varepsilon e^{\varepsilon t} x^{T}(t) G x(t) d t \\
& +e^{\varepsilon t} d\left[x^{T}(t) G x(t)\right], \quad \text { for any } t \geq 0 .
\end{aligned}
$$


Substituting (23) into (25) yields

$$
\begin{aligned}
& e^{\varepsilon t} x^{T}(t) G x(t) \\
& \leq \xi^{T}(0) G \xi(0)+\varepsilon \int_{0}^{t} e^{\varepsilon s} x^{T}(s) G x(s) d s \\
& \quad-\int_{0}^{t} e^{\varepsilon s}\left[\lambda_{\min }(Q)-\|G\|\left(2 \alpha_{1}+2 \alpha_{2}+\beta_{1}+\beta_{2}+\alpha_{3}\right)-\beta\right] \\
& \cdot|x(s)|^{2} d s+\int_{0}^{t} e^{\varepsilon s} \cdot\|G\|\left(\beta_{1}+\beta_{2}+\beta_{3}\right)|x(s-\tau)|^{2} d s \\
& +\int_{0}^{t} e^{\varepsilon s} \cdot \frac{1}{\beta}\|G B\|^{2} \cdot|x(s)-x(s-\tau)|^{2} d s \\
& +\int_{0}^{t} e^{\varepsilon s} \cdot 2 x^{T}(s) G h(s, x(s), x(s-\tau)) d W(s) .
\end{aligned}
$$

Taking the expectation in (26), we have

$$
\begin{aligned}
& E\left(e^{\varepsilon t} x^{T}(t) G x(t)\right) \\
& \leq E\left(\xi^{T}(0) G \xi(0)\right) \\
&-\left[\lambda_{\min }(Q)-\|G\|\left(2 \alpha_{1}+2 \alpha_{2}+\beta_{1}+\beta_{2}+\alpha_{3}+\varepsilon\right)-\beta\right] \\
& \cdot \int_{0}^{t} e^{\varepsilon s} \cdot E|x(s)|^{2} d s \\
&+\|G\|\left(\beta_{1}+\beta_{2}+\beta_{3}\right) \int_{0}^{t} e^{\varepsilon s} \cdot E|x(s-\tau)|^{2} d s \\
&+\frac{1}{\beta}\|G B\|^{2} \int_{0}^{t} e^{\varepsilon s} \cdot E|x(s)-x(s-\tau)|^{2} d s .
\end{aligned}
$$

In order to give an estimate of $E\left(e^{\varepsilon t} x^{T}(t) G x(t)\right)$, we now estimate the last two terms on the right-hand side of (27). First of all, for any $t \geq \tau$, we have

$$
\begin{aligned}
\int_{0}^{t} & e^{\varepsilon s} \cdot E|x(s-\tau)|^{2} d s \\
& =\int_{0}^{\tau} e^{\varepsilon s} \cdot E|x(s-\tau)|^{2} d s+\int_{\tau}^{t} e^{\varepsilon s} \cdot E|x(s-\tau)|^{2} d s \\
& \leq e^{\varepsilon \tau} \cdot \int_{0}^{\tau} E|\xi(s-\tau)|^{2} d s+e^{\varepsilon \tau} \cdot \int_{\tau}^{t} e^{\varepsilon(s-\tau)} \cdot E|x(s-\tau)|^{2} d s \\
= & e^{\varepsilon \tau} \cdot \int_{-\tau}^{0} E|\xi(u)|^{2} d u+e^{\varepsilon \tau} \\
& \cdot \int_{0}^{t-\tau} e^{\varepsilon u} \cdot E|x(u)|^{2} d u \quad(u=s-\tau) \\
= & c_{1} e^{\varepsilon \tau}+e^{\varepsilon \tau} \cdot \int_{0}^{t-\tau} e^{\varepsilon s} \cdot E|x(s)|^{2} d s,
\end{aligned}
$$

where $c_{1}=\int_{-\tau}^{0} E|x(s)|^{2} d s$.
Next, recalling (14) and (15), for $s \geq \tau$, we derive

$$
\begin{aligned}
& E|x(s)-x(s-\tau)|^{2} \\
& \leq 2 \tau \cdot E \int_{s-\tau}^{s} 6\left[\left(\|A\|^{2}+\alpha_{1}^{2}+\alpha_{2}^{2}\right)|x(t)|^{2}\right. \\
& \left.\quad+\left(\|B\|^{2}+\beta_{1}^{2}+\beta_{2}^{2}\right)|x(t-\tau)|^{2}\right] d t \\
& \quad+2 E \int_{s-\tau}^{s}\left(\alpha_{3}|x(t)|^{2}+\beta_{3} x(t-\tau)^{2}\right) d t \\
& \leq 2\left[6 \tau\left(\|A\|^{2}+\alpha_{1}^{2}+\alpha_{2}^{2}\right)+\alpha_{3}\right] \int_{s-\tau}^{s} E|x(t)|^{2} d t \\
& \quad+2\left[6 \tau\left(\|B\|^{2}+\beta_{1}^{2}+\beta_{2}^{2}\right)+\beta_{3}\right] \int_{s-\tau}^{s} E|x(t-\tau)|^{2} d t .
\end{aligned}
$$

Similar to (28), for $t \geq \tau$, we have

$$
\begin{aligned}
\int_{0}^{t} e^{\varepsilon s} & \cdot E|x(s)-x(s-\tau)|^{2} d s \\
= & \int_{0}^{\tau} e^{\varepsilon s} \cdot E|x(s)-x(s-\tau)|^{2} d s \\
& +\int_{\tau}^{t} e^{\varepsilon s} \cdot E|x(s)-x(s-\tau)|^{2} d s \\
\leq & c_{2}+2\left[6 \tau\left(\|A\|^{2}+\alpha_{1}^{2}+\alpha_{2}^{2}\right)+\alpha_{3}\right] \\
& \cdot \int_{\tau}^{t} e^{\varepsilon s} \int_{s-\tau}^{s} E|x(\nu)|^{2} d \nu d s \\
& +2\left[6 \tau\left(\|B\|^{2}+\beta_{1}^{2}+\beta_{2}^{2}\right)+\beta_{3}\right] \\
& \cdot \int_{\tau}^{t} e^{\varepsilon s} \int_{s-\tau}^{s} E|x(\nu-\tau)|^{2} d \nu d s,
\end{aligned}
$$

where $c_{2}=\int_{0}^{\tau} e^{\varepsilon s} \cdot E|x(s)-x(s-\tau)|^{2} d s$. Moreover,

$$
\begin{aligned}
\int_{\tau}^{t} e^{\varepsilon s} \int_{s-\tau}^{s} E|x(\nu)|^{2} d \nu d s & =\int_{0}^{t} E|x(\nu)|^{2}\left(\int_{\nu \vee \tau}^{(\nu+\tau) \wedge t} e^{\varepsilon s} d s\right) d \nu \\
& \leq \tau e^{\varepsilon \tau} \int_{0}^{t} e^{\varepsilon \nu} \cdot E|x(\nu)|^{2} d \nu .
\end{aligned}
$$

Similarly,

$$
\begin{aligned}
\int_{\tau}^{t} e^{\varepsilon s} & \int_{s-\tau}^{s} E|x(\nu-\tau)|^{2} d \nu d s \\
\quad & \int_{0}^{t} E|x(\nu-\tau)|^{2}\left(\int_{\nu \vee \tau}^{(\nu+\tau) \wedge t} e^{\varepsilon s} d s\right) d \nu \\
& \leq \tau e^{\varepsilon \tau} \int_{0}^{t} e^{\varepsilon \nu} \cdot E|x(\nu-\tau)|^{2} d \nu
\end{aligned}
$$


Substituting (28) into (32) yields

$$
\begin{aligned}
\int_{\tau}^{t} e^{\varepsilon s} & \int_{s-\tau}^{s} E|x(\nu-\tau)|^{2} d \nu d s \\
& \leq \tau e^{\varepsilon \tau}\left(c_{1} e^{\varepsilon \tau}+e^{\varepsilon \tau} \cdot \int_{0}^{t-\tau} e^{\varepsilon s} \cdot E|x(s)|^{2} d s\right) \\
& <\tau c_{1} e^{2 \varepsilon \tau}+\tau e^{2 \varepsilon \tau} \int_{0}^{t} e^{\varepsilon s} \cdot E|x(s)|^{2} d s .
\end{aligned}
$$

Substituting (31) and (33) into (30), for $t \geq \tau$, we get

$$
\begin{aligned}
\int_{0}^{t} e^{\varepsilon s} & \cdot E|x(s)-x(s-\tau)|^{2} d s \\
\leq & c_{2}+2\left[6 \tau\left(\|A\|^{2}+\alpha_{1}^{2}+\alpha_{2}^{2}\right)+\alpha_{3}\right] \\
\cdot & \tau e^{\varepsilon \tau} \int_{0}^{t} e^{\varepsilon v} \cdot E|x(\nu)|^{2} d \nu \\
+ & 2\left[6 \tau\left(\|B\|^{2}+\beta_{1}^{2}+\beta_{2}^{2}\right)+\beta_{3}\right] \\
& \cdot\left(\tau c_{1} e^{2 \varepsilon \tau}+\tau e^{2 \varepsilon \tau} \int_{0}^{t} e^{\varepsilon s} \cdot E|x(s)|^{2} d s\right) .
\end{aligned}
$$

Substituting (28) and (34) into (27) and recalling (24), we obtain that for $t \geq \tau$

$$
\begin{gathered}
E\left(e^{\varepsilon t} x^{T}(t) G x(t)\right) \\
\leq E\left(\xi^{T}(0) G \xi(0)\right) \\
-\left[\lambda_{\min }(Q)-\|G\|\left(2 \alpha_{1}+2 \alpha_{2}+\beta_{1}+\beta_{2}+\alpha_{3}+\varepsilon\right)-\beta\right] \\
\cdot \int_{0}^{t} e^{\varepsilon s} \cdot E|x(s)|^{2} d s \\
+\|G\|\left(\beta_{1}+\beta_{2}+\beta_{3}\right)\left(c_{1} e^{\varepsilon \tau}+e^{\varepsilon \tau} \cdot \int_{0}^{t-\tau} e^{\varepsilon s} \cdot E|x(s)|^{2} d s\right) \\
+\frac{1}{\beta}\|G B\|^{2}\left\{c_{2}+2\left[6 \tau\left(\|A\|^{2}+\alpha_{1}^{2}+\alpha_{2}^{2}\right)+\alpha_{3}\right]\right. \\
\quad \cdot \tau e^{\varepsilon \tau} \int_{0}^{t} e^{\varepsilon s} \cdot E|x(s)|^{2} d s \\
+2\left[6 \tau\left(\|B\|^{2}+\beta_{1}^{2}+\beta_{2}^{2}\right)+\beta_{3}\right] \\
\left.\quad\left(\tau c_{1} e^{2 \varepsilon \tau}+\tau e^{2 \varepsilon \tau} \int_{0}^{t} e^{\varepsilon s} \cdot E|x(s)|^{2} d s\right)\right\}
\end{gathered}
$$$$
=c_{3} \text {, }
$$

where

$$
\begin{array}{rl}
c_{3} & E\left(\xi^{T}(0) G \xi(0)\right)+\|G\|\left(\beta_{1}+\beta_{2}+\beta_{3}\right) \cdot c_{1} e^{\varepsilon \tau} \\
& +\frac{1}{\beta}\|G B\|^{2}\left\{c_{2}+2\left[6 \tau\left(\|B\|^{2}+\beta_{1}^{2}+\beta_{2}^{2}\right)+\beta_{3}\right] \cdot \tau c_{1} e^{2 \mathcal{E} \tau}\right\} .
\end{array}
$$

Since $G$ is positive definite,

$$
x^{T}(t) G x(t) \geq \lambda_{\min }(G)|x(t)|^{2},
$$

where $\lambda_{\min }(G)>0$ is the smallest eigenvalue of $G$.

Thus,

$$
E\left(e^{\varepsilon t} x^{T}(t) G x(t)\right) \geq E\left(e^{\varepsilon t} \lambda_{\min }(G)|x(t)|^{2}\right) .
$$

It then follows from (35) that

$$
E|x(t)|^{2} \leq \frac{c_{3}}{\lambda_{\min }(G)} \cdot e^{-\varepsilon t} \quad \text { for any } t \geq \tau
$$

Hence,

$$
\begin{aligned}
\frac{1}{t} \ln \left(E|x(t)|^{2}\right) & \leq \frac{1}{t} \ln \left(\frac{c_{3}}{\lambda_{\min }(G)} \cdot e^{-\varepsilon t}\right) \\
& =-\varepsilon+\frac{1}{t} \ln \left(\frac{c_{3}}{\lambda_{\min }(G)}\right) .
\end{aligned}
$$

This easily yields

$$
\limsup _{t \rightarrow \infty} \frac{1}{t} \ln \left(E|x(t)|^{2}\right) \leq-\varepsilon
$$

Then, (13) is exponentially stable in the mean square. The proof is complete.

Remark 6. In the proof we gave, the estimate for the second moment Lyapunov exponent should not be greater than $-\varepsilon$.

Theorem 7. The trivial solution of system (13) is also almost surely exponentially stable under the same assumption as Theorem 5.

Proof. For $t \in[k \tau,(k+1) \tau], k=2,3, \ldots$, we have

$$
\begin{aligned}
& |x(t)|^{2} \\
& \quad \leq 3|x(k \tau)|^{2} \\
& \quad+3 \mid \int_{k \tau}^{(k+1) \tau}\left[\left(A+A_{1}(t)+A_{2}(t-\tau)\right) x(t)\right. \\
& \left.+\left(B+B_{1}(t)+B_{2}(t-\tau)\right) x(t-\tau)\right]\left.d t\right|^{2} \\
& +3\left|\int_{k \tau}^{(k+1) \tau} h(t, x(t), x(t-\tau)) d W(t)\right|^{2} .
\end{aligned}
$$


Recalling (14) and (15), we derive

$$
\begin{aligned}
& E\left(\sup _{k \tau \leq t \leq(k+1) \tau}|x(t)|^{2}\right) \\
& \leq 3 E|x(k \tau)|^{2} \\
& \quad+18 \tau \int_{k \tau}^{(k+1) \tau}\left[\left(\|A\|^{2}+\alpha_{1}^{2}+\alpha_{2}^{2}\right) E|x(t)|^{2}\right. \\
& \left.\quad+\left(\|B\|^{2}+\beta_{1}^{2}+\beta_{2}^{2}\right) E|x(t-\tau)|^{2}\right] d t \\
& \quad+3 \int_{k \tau}^{(k+1) \tau}\left[\alpha_{3} E|x(t)|^{2}+\beta_{3} E|x(t-\tau)|^{2}\right] d t .
\end{aligned}
$$

By (39), we easily get

$$
\begin{gathered}
E|x(k \tau)|^{2} \leq \frac{c_{3}}{\lambda_{\min }(G)} \cdot e^{-\varepsilon k \tau} \quad \text { for } k \tau \geq \tau, \\
E|x(t-\tau)|^{2} \leq \frac{c_{3}}{\lambda_{\min }(G)} \cdot e^{-\varepsilon(t-\tau)} \quad \text { for } t-\tau \geq \tau .
\end{gathered}
$$

Substituting the above two into (43) yields

$$
\begin{aligned}
& E\left(\sup _{k \tau \leq t \leq(k+1) \tau}|x(t)|^{2}\right) \\
& \leq \frac{3 c_{3}}{\lambda_{\min }(G)}\left\{e^{-\varepsilon k \tau}+\left[6 \tau\left(\|A\|^{2}+\alpha_{1}^{2}+\alpha_{2}^{2}\right)+\alpha_{3}\right]\right. \\
& \int_{k \tau}^{(k+1) \tau} e^{-\varepsilon t} d t+\left[6 \tau\left(\|B\|^{2}+\beta_{1}^{2}+\beta_{2}^{2}\right)+\beta_{3}\right] \\
& \left.\cdot e^{\varepsilon \tau} \int_{k \tau}^{(k+1) \tau} e^{-\varepsilon t} d t\right\} \\
& \leq \frac{3 c_{3}}{\lambda_{\min }(G)}\left\{e^{-\varepsilon k \tau}+\left(\left[6 \tau\left(\|A\|^{2}+\alpha_{1}^{2}+\alpha_{2}^{2}\right)+\alpha_{3}\right]\right.\right. \\
& \left.+\left[6 \tau\left(\|B\|^{2}+\beta_{1}^{2}+\beta_{2}^{2}\right)+\beta_{3}\right] e^{\varepsilon \tau}\right) \\
& \left.\cdot \frac{1}{\mathcal{\varepsilon}} e^{-\varepsilon k \tau}\left(1-e^{-\varepsilon \tau}\right)\right\} \\
& \leq \frac{3 c_{3}}{\lambda_{\min }(G)}\left\{e^{-\varepsilon k \tau}+\left(\left[6 \tau\left(\|A\|^{2}+\alpha_{1}^{2}+\alpha_{2}^{2}\right)+\alpha_{3}\right]\right.\right. \\
& \left.\left.+\left[6 \tau\left(\|B\|^{2}+\beta_{1}^{2}+\beta_{2}^{2}\right)+\beta_{3}\right] e^{\varepsilon \tau}\right) \tau e^{-\varepsilon k \tau}\right\} \\
& =\frac{3 \tau c_{3}}{\lambda_{\min }(G)}\left\{\frac{1}{\tau}+\left[6 \tau\left(\|A\|^{2}+\alpha_{1}^{2}+\alpha_{2}^{2}\right)+\alpha_{3}\right]\right. \\
& \left.+\left[6 \tau\left(\|B\|^{2}+\beta_{1}^{2}+\beta_{2}^{2}\right)+\beta_{3}\right] e^{\varepsilon \tau}\right\} \cdot e^{-\varepsilon k \tau} \\
& =c_{4} e^{-\varepsilon k \tau}
\end{aligned}
$$

where $c_{4}=\left(3 \tau c_{3} / \lambda_{\min }(G)\right)\left\{(1 / \tau)+\left[6 \tau\left(\|A\|^{2}+\alpha_{1}^{2}+\alpha_{2}^{2}\right)+\alpha_{3}\right]+\right.$ $\left.\left[6 \tau\left(\|B\|^{2}+\beta_{1}^{2}+\beta_{2}^{2}\right)+\beta_{3}\right] e^{\varepsilon \tau}\right\}$.
Let $\varepsilon_{0} \in(0, \varepsilon)$ be arbitrary. By Doob's martingale inequality, it follows from (45) that

$$
P\left(\omega: \sup _{k \tau \leq t \leq(k+1) \tau}|x(t)|>e^{-\left(\varepsilon-\varepsilon_{0}\right) k \tau / 2}\right) \leq c_{4} e^{-\varepsilon_{0} k \tau} .
$$

It then follows from the Borel-Cantelli lemma that for almost all $\omega \in \Omega$, there exists a $k_{0}(\omega), k \geq k_{0}(\omega)$, and

$$
\sup _{k \tau \leq t \leq(k+1) \tau}|x(t)| \leq e^{-\left(\varepsilon-\varepsilon_{0}\right) k \tau / 2}
$$

holds in probability 1 . Thus, for $k \tau \leq t \leq(k+1) \tau$, and $k \geq$ $k_{0}(\omega)$, we have

$$
\ln |x(t)| \leq-\frac{\left(\varepsilon-\varepsilon_{0}\right) k \tau}{2} \quad \text { a.s. }
$$

Hence,

$$
\frac{1}{t} \ln |x(t)| \leq-\frac{\varepsilon-\varepsilon_{0}}{2} \quad \text { a.s. }
$$

This easily yields

$$
\limsup _{t \rightarrow \infty} \frac{1}{t} \ln |x(t)| \leq-\frac{\varepsilon-\varepsilon_{0}}{2} \quad \text { a.s. }
$$

Since $\varepsilon_{0}$ is arbitrary, we must have

$$
\limsup _{t \rightarrow \infty} \frac{1}{t} \ln |x(t)| \leq-\frac{\varepsilon}{2} \quad \text { a.s. }
$$

Then, (13) is almost surely exponentially stable. The proof is complete.

Remark 8. Again in the proof we gave, the estimate for the almost surely Lyapunov exponent should not be greater than $-\varepsilon / 2$.

Let us single out three important special cases.

Case 1. If $h(t, x(t), x(t-\tau)) \equiv 0$, then (13) reduces to a semilinear differential delay equation

$$
\begin{aligned}
d x(t)=[ & \left(A+A_{1}(t)+A_{2}(t-\tau)\right) x(t) \\
& \left.+\left(B+B_{1}(t)+B_{2}(t-\tau)\right) x(t-\tau)\right] d t
\end{aligned}
$$

Corollary 9. Let condition (14) holds. Assume that there exists a pair of symmetric positive definite $n \times n$ matrices $G$ and $Q$ such that

$$
\begin{gathered}
G(A+B)+(A+B)^{T} G=-Q, \\
\lambda_{\min }(Q) \\
>2\|G\|\left(\alpha_{1}+\alpha_{2}+\beta_{1}+\beta_{2}\right) \\
+4 \tau\|G B\| \cdot \sqrt{3\left(\|A\|^{2}+\|B\|^{2}+\alpha_{1}^{2}+\beta_{1}^{2}+\alpha_{2}^{2}+\beta_{2}^{2}\right)} .
\end{gathered}
$$

Then, (52) is exponentially stable in the mean square. 
Case 2. Let us further assume that $A_{1}(t)=A_{2}(t-\tau)=$ $B_{1}(t)=B_{2}(t-\tau) \equiv 0$, then (52) becomes an ordinary differential equation

$$
d x(t)=(A x(t)+B x(t-\tau)) d t .
$$

Corollary 10. Assume that there exists a pair of symmetric positive definite $n \times n$ matrices $G$ and $Q$ such that

$$
\begin{gathered}
G(A+B)+(A+B)^{T} G=-Q, \\
\lambda_{\min }(Q)>4 \tau\|G B\| \cdot \sqrt{3\left(\|A\|^{2}+\|B\|^{2}\right)} .
\end{gathered}
$$

Then, (55) is exponentially stable in the mean square.

Remark 11. Corollary 10 clearly shows that if the price system $\dot{x}(t)=(A+B) x(t)$ (the general case of the classical price Rayleigh equation (2)) is exponentially stable (this is guaranteed by condition (16) and $\lambda_{\min }(Q)>0$ ), then the corresponding delayed system (55) is also exponentially stable provided that the time delay $\tau$ is small enough (bounded by (57)).

Remark 12. Condition (17) controls the intensity of the uncertainties, that is, the parameters $\alpha_{i}, \beta_{i}(i=1,2,3)$, and the time delay $\tau$ should be small enough to have the stability of (13) which is regarded as the perturbed system of (55). In other words, Theorem 5 shows that if (55) is exponentially stable, so is its perturbed system (13) provided that the intensity of perturbations is small enough.

Case 3. Let us assume that $B=A_{2}(t-\tau)=B_{1}(t)=B_{2}(t-$ $\tau) \equiv 0$ and $\tau \equiv 0$, then (13) reduces to a stochastic differential equation

$$
d x(t)=\left(A+A_{1}(t)\right) x(t) d t+h(t, x(t)) d W(t) .
$$

Corollary 13. Assume that there exists a pair of symmetric positive definite $n \times n$ matrices $G$ and $Q$ such that

$$
\begin{gathered}
G A+A^{T} G=-Q, \\
\lambda_{\min }(Q)>\|G\|\left(2 \alpha_{1}+\alpha_{3}\right) .
\end{gathered}
$$

Then, (58) is exponentially stable in the mean square and is also almost surely exponentially stable.

\section{Example}

Let us now present two simple examples to illustrate our results which can help us find the time delay upper limit.

Example 14. Let us start with (13), where $\alpha_{1}=\alpha_{2}=\alpha_{3}=\beta_{1}=$ $\beta_{2}=\beta_{3}=0.1$,

$$
A=\left[\begin{array}{cc}
-1 & 0 \\
-1 & -1
\end{array}\right], \quad B=\left[\begin{array}{cc}
-2 & 0 \\
1 & -1
\end{array}\right]
$$

For convenience, let us choose $Q=$ the 2-order identity matrix, and then $\lambda_{\text {min }}(Q)=1$. By plugging these into (16), it is easy to find that

$$
G=\left[\begin{array}{ll}
\frac{1}{6} & 0 \\
0 & \frac{1}{4}
\end{array}\right] .
$$

We obtain via a simple calculation

$$
\begin{aligned}
\|A\|^{2}=2.618, \quad\|B\|^{2} & =5.2361, \quad\|G\|=0.25, \\
\|G B\| & =0.4488
\end{aligned}
$$

Substituting (63) into (17), we derive that if $\tau<0.08380$, then (13) is exponentially stable in the mean square and is also almost surely exponentially stable. If $h(t, x(t), x(t-\tau)) \equiv 0$, by Corollary 9 , we would conclude that (52) is exponentially stable provided that $\tau<0.09156$.

Example 15. Now, let us recall price system (4) with $a=$ $-0.5, b_{0}=1.0, c_{0}=1.0, \gamma=0.2, \varepsilon=0.1, \sqrt{\lambda \alpha}=1$, and $\tilde{\mu}=0.06$. It is clear that $z(t)=(0,0)^{T}$ is the trivial solution of system (4). Applying Taylor expansion to $f(t, z(t))$, we get

$$
f(t, z(t))=f(t, 0)+A z(t)+A_{1}(t) z(t),
$$

where $A=\left[\begin{array}{cc}0.006 & 1 \\ -1 & 0\end{array}\right], \operatorname{tr}\left[h^{T}(t, x(t)) h(t, x(t))\right]=0.004$, and $\left\|A_{1}(t)\right\| \leq 0.001$.

To compute conveniently, let us choose $\alpha_{3}=0.004$ and $Q=\left[\begin{array}{cc}0.01 & 0 \\ 0 & 0.01\end{array}\right]$, and then $\lambda_{\min }(Q)=0.01$. By Corollary 13 , it is easy to find

$$
G=\left[\begin{array}{cc}
1.66667 & -0.00500 \\
-0.00500 & 1.66670
\end{array}\right], \quad\|G\|=0.5285
$$

Substituting (65) into (60), it is easy to verify that (60) holds. So, the price system (4) with $a=-0.5, b_{0}=1.0, c_{0}=1.0, \gamma=$ $0.2, \varepsilon=0.1, \sqrt{\lambda \alpha}=1$, and $\tilde{\mu}=0.06$ is exponentially stable in the mean square and is also almost surely exponentially stable. This result is the same as [5].

\section{Concluding Remarks}

In this paper, we study the exponential stability of the stochastic nonlinear dynamical price system. Using Taylor's theorem, the stochastic nonlinear system with delay is reduced to an $n$ dimensional semilinear stochastic differential equation with delay. Some sufficient conditions of exponential stability and corollaries for the price system are established by virtue of Lyapunov function. The time delay upper limit is solved by using our theoretical results when the system is exponentially stable, and [5] is promoted and improved. Remarks 11 and 12 show that if price Rayleigh equation (2) is exponentially stable, so is its perturbed system (13) provided that both the time delay and the intensity of perturbations are small enough. These results are very helpful for our government strengthening and improving macrocontrol and promoting 
steady and rapid economic development. It is also an important guiding significance that our government can timely adjust their pricing strategies. Two examples are presented to illustrate our theoretical results, which are the same as [5]. Another challenging problem is to study a type of stochastic nonlinear dynamical price system with variable delay. We hope to study these problems in forthcoming papers.

\section{Acknowledgments}

This work is Supported by the Fundamental Research Funds for the Central Universities (JBK130213) and the Fundamental Research Funds for the Central Universities (JBK130401).

\section{References}

[1] S. Wang, Differential Equation Model and Chaos, China Science and Technology University Press, Hefei, China, 2002.

[2] Y. Liu and Z. Feng, "Stochastic stability \& control," in Large Power System Theory and Application, South China University of Science and Technology Press, Guangzhou, China, 4th edition, 1992.

[3] Y. Liu and F. Deng, "Random system variable structure system," in Large Power System Theory and Application, South China University of Science and Technology Press, Guangzhou, China, 10th edition, 1992.

[4] J. Yong and X. Y. Zhou, Stochastic Controls: Hamiltonian Systems and HJB Equations, vol. 43 of Applications of Mathematics, Springer, New York, NY, USA, 1999.

[5] J. R. Li and W. Xu, "Optimal control of a stochastic nonlinear dynamical price model," Pure and Applied Mathematics, vol. 24, no. 2, pp. 239-244, 2008.

[6] Y. Kazmerchuk, A. Swishchuk, and J. Wu, "The pricing of options for securities markets with delayed response," Mathematics and Computers in Simulation, vol. 75, no. 3-4, pp. 69-79, 2007.

[7] T. Lv and Z. W. Liu, "Hopf bifurcation of price Rayleigh equations with delay," Journal of Jilin University, vol. 47, no. 3 , pp. 441-448, 2009.

[8] T. Lv and L. Zhou, "Hopf and codimension two bifurcation in price Rayleigh equation with two time delay," Journal of Jilin University, vol. 50, no. 3, pp. 441-448, 2012.

[9] X. Zhang, X. Chen, and Y. Chen, "A qualitative analysis of price model in differential equations of price," Journal of Shenyang Institute of Aeronautical Engineering, vol. 21, no. 1, pp. 83-86, 2004.

[10] X. Mao, Exponential Stability of Stochastic Differential Equations, vol. 182 of Monographs and Textbooks in Pure and Applied Mathematics, Marcel Dekker, New York, NY, USA, 1994.

[11] W. Zhu, J. Hu, and J. Zhang, "Stability analysis of neural networks with time delays via energy functions approach," in Proceedings of the 7th International Conference on Natural Computation (ICNC '11), vol. 1, pp. 232-236, 2011.

[12] W. Zhu and Z. Yi, "Integral input-to-state stability of nonlinear control systems with delays," Chaos, Solitons \& Fractals, vol. 34, no. 2, pp. 420-427, 2007.

[13] H. Trinh and M. Aldeen, "On robustness and stabilization of linear systems with delayed nonlinear perturbations," IEEE Transactions on Automatic Control, vol. 42, no. 7, pp. 1005-1007, 1997.
[14] X. Mao, "Robustness of exponential stability of stochastic differential delay equations," IEEE Transactions on Automatic Control, vol. 41, no. 3, pp. 442-447, 1996.

[15] X. Mao and A. Shah, "Exponential stability of stochastic differential delay equations," Stochastics and Stochastics Reports, vol. 60, no. 1-2, pp. 135-153, 1997.

[16] W. Zhu and J. Hu, "Stability analysis of stochastic delayed cellular neural networks by LMI approach," Chaos, Solitons \& Fractals, vol. 29, no. 1, pp. 171-174, 2006.

[17] W. Zhu and J. Hu, "Exponential stability of stochastic recurrent neural network with time delays," International Journal of Computational Intelligence Research, vol. 2, no. 1, pp. 52-54, 2006.

[18] S. Xie and L. Xie, "Stabilization of a class of uncertain large-scale stochastic systems with time delays," Automatica, vol. 36, no. 1, pp. 161-167, 2000.

[19] B. Øksendal, Stochastic Differential Equations: An Introduction with Applications, Springer, Berlin, Germany, 5th edition, 1998.

[20] W. Zhu, J. Hu, P. Li, and C. Zhu, "On the energy functions of neural networks with delay," Dynamics of Continuous, Discrete \& Impulsive Systems B, vol. 18, no. 5, pp. 589-599, 2011. 


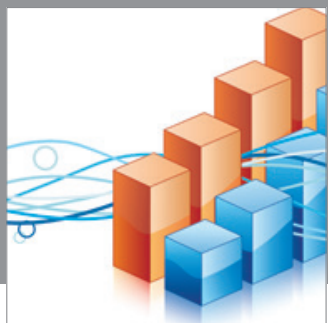

Advances in

Operations Research

mansans

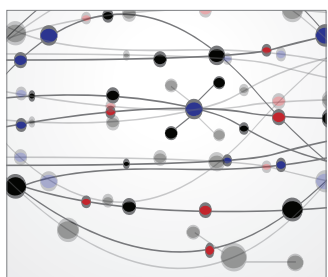

The Scientific World Journal
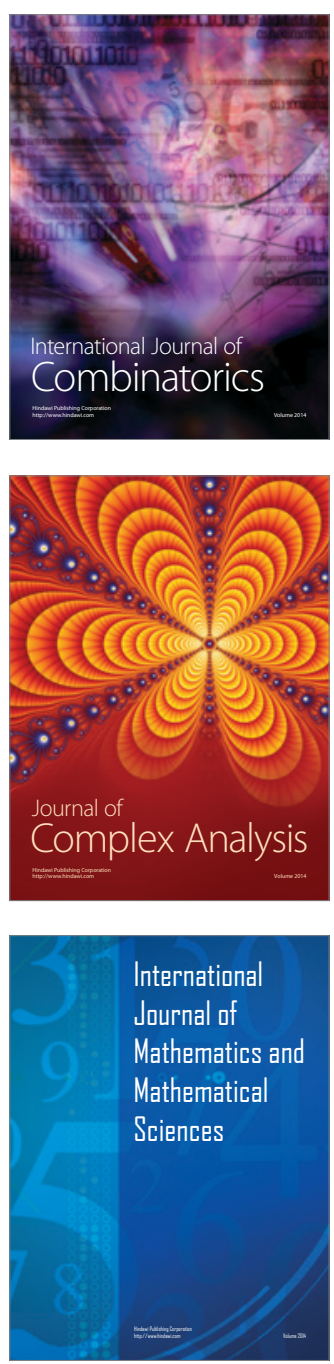
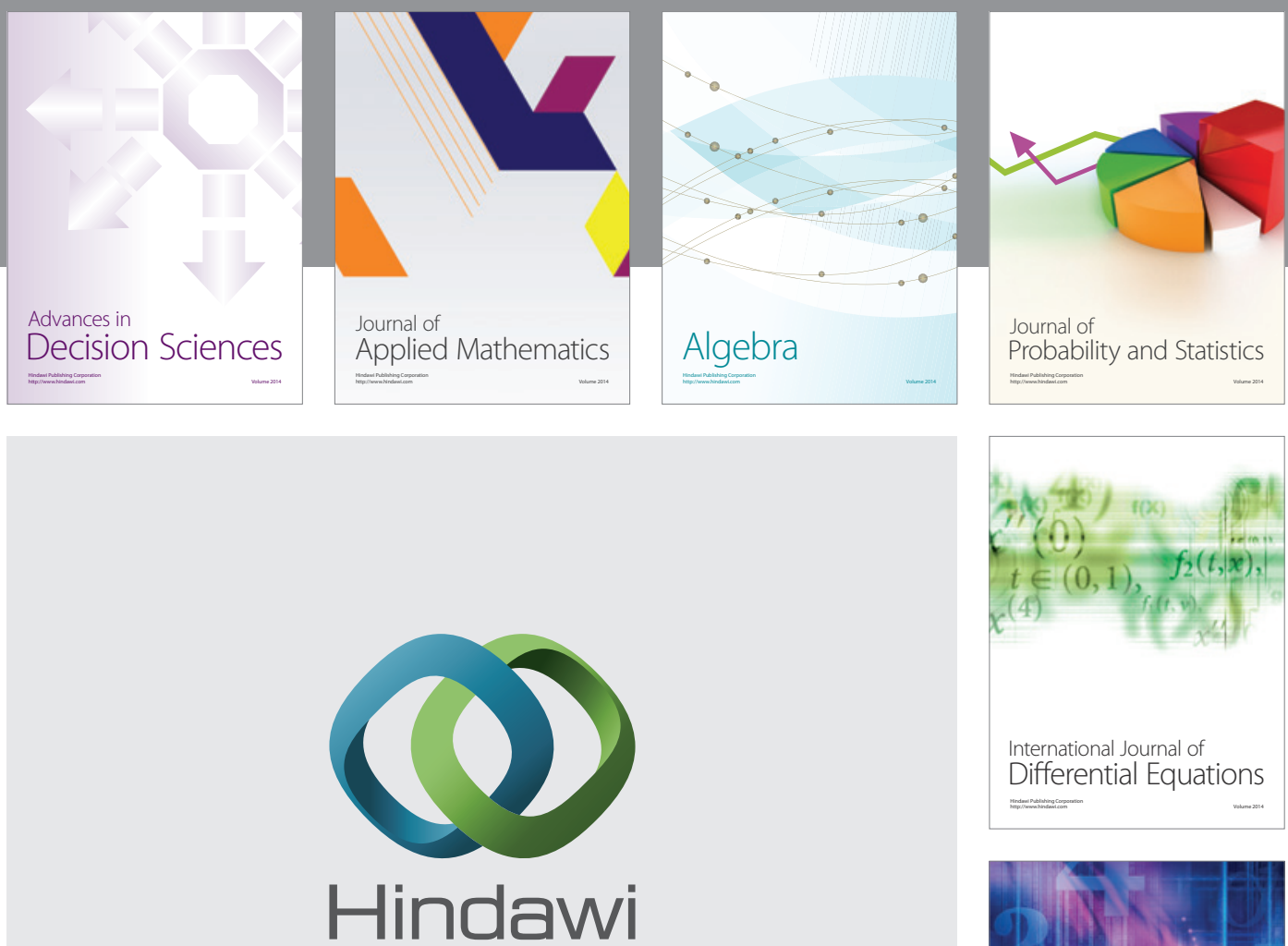

Submit your manuscripts at http://www.hindawi.com
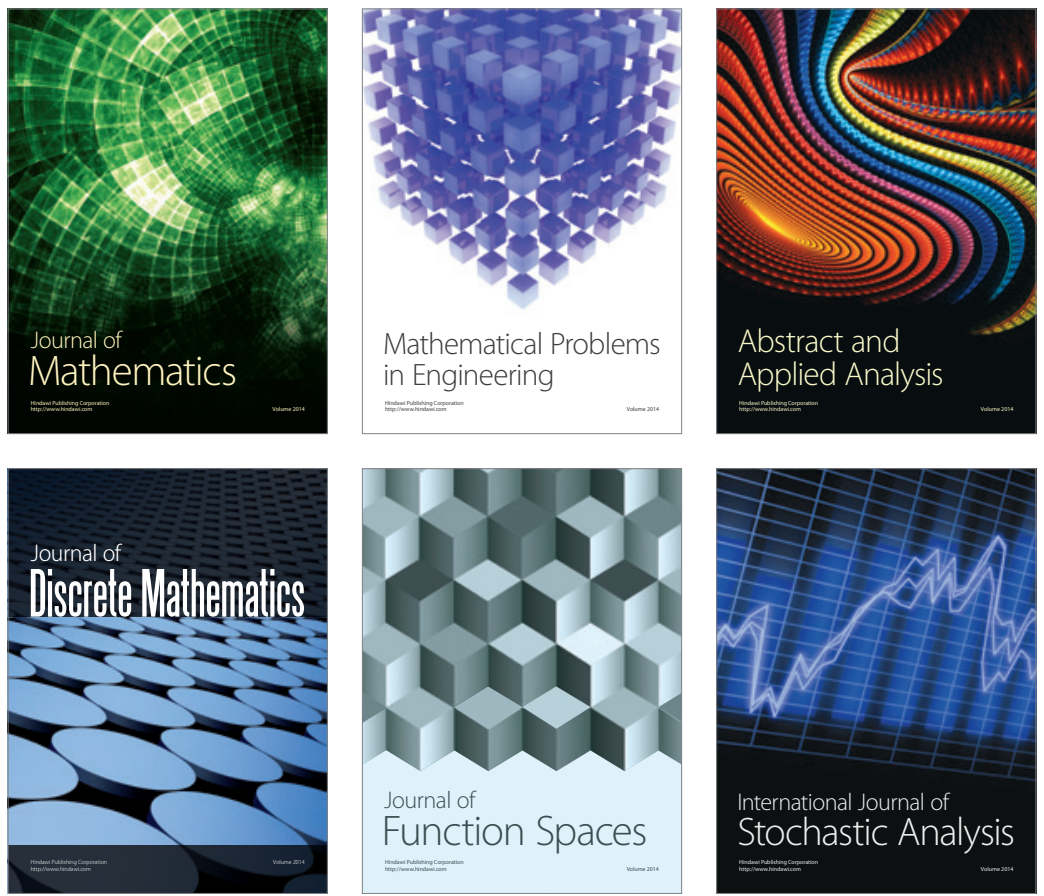

Journal of

Function Spaces

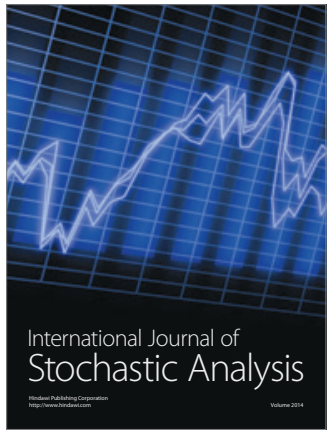

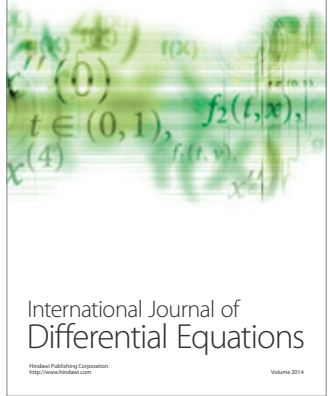
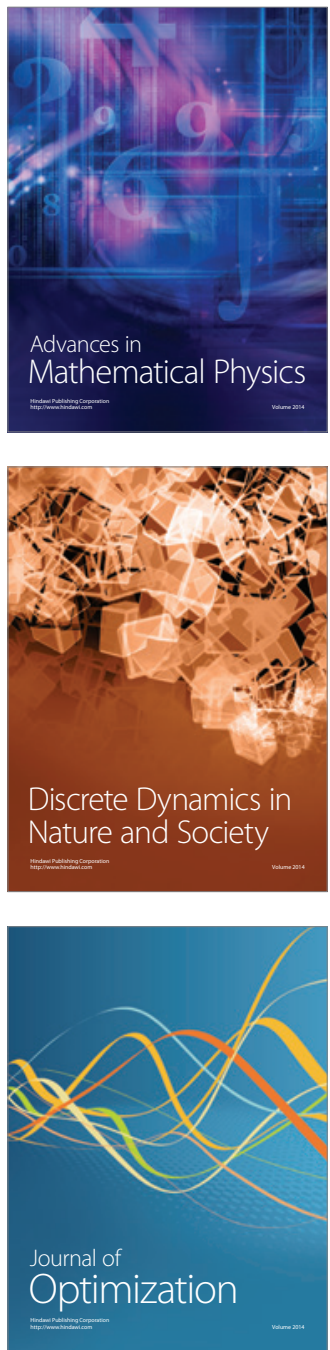